\title{
SUR L'APPAREIL DE SOUTIEN DANS LES TIGES DES SAXIFRAGES,
} par M. THOUVENIN.

Il est admis, depuis les travaux de M. Costantin, que l'appareil de soutien, généralement bien développé dans les tiges aériennes et dressées, est ordinairement nul ou très réduit dans les tiges végétant horizontalement, tant souterraines que rampant à la surface du sol.

Pour les Saxifrages en particulier, M. Costantin a établi que, dans les tiges souterraines, le stéréome, qui dans les tiges aériennes était représenté par le péricycle devenu scléreux, disparaît complètement; réserve faite, toutefois, des vaisseaux qui, dans une certaine mesure, font partie de l'appareil de soutien.

I. Costantin, dont les recherches s'étendaient à un grand nombre de familles, n'a pu examiner, en fait de Saxifrages, que les Saxifraga stellaris, Aizoon et aizoides, et c'est de leur étude qu'il tire ses conclusions sur ce genre (1).

Mes recherches personnelles, ayant porté sur un assez grand nombre d'espèces de Saxifrages, m'ont fait voir que, si dans les tiges couchées l'anneau de soutien dù au péricycle sclérifié disparaît, ces tiges n'en sont pas moins pourvues, dans un certain nombre d'espèces, d'un stéréome assez puissant.

Chez les unes, c'est le péricycle qui avec l'anneau externe de la moelle est devenu collenchymateux et forme, par conséquent, un appareil de soutien; dans d'autres, cet appareil est encore bien développé, mais s'est constitué par la sclérose d'autres tissus que le péricycle.

Si les tiges aériennes, ou plutôt les hampes florales, ont une grande uniformité de structure dans le genre Saxifrage, il n'en est donc pas de mème pour les tiges rampantes et souterraines.

Aussi est-il avantageux, pour l'étude de ces tiges, de grouper, autour d'un certain nombre de types, les tiges des différentes espèces de ce grand genre. Étudier les différentes manières d'être du stéréome, dans les tiges couchées des Saxifrages, tel est principalement l'objet de celte Note.

TYPE I. Saxifraga stellaris L. - Dans la tige souterraine, l'appareil de soutien est aussi réduit que possible.

L'écorce, le péricycle et la moelle sont composés, uniquement, d'éléments parenchymateux; les faisceaux libéro-ligneux, confluents en un

(1) Costantin, Et. comp. des tiges aer. et souterr. des Dicotylédones (Ann. des sc. nat., Ge série, t. XVI, p. 79). 
cercle continu, ont le bois formé de vaisseaux disséminés dans un parenchyme ligneux abondant.

Les faisceaux, dans la tige aérienne, s'individualisent; le péricycle devient scléreux et les cellules de l'endoderme épaississent et lignifient légèrement leurs parois de façon à faire partie, elles aussi, de l'appareil de soutien.

Entre ces deux sortes de tiges de structure si différente il y a une portion intermédiaire, dont l'étude montre comment s'opère le passage de la structure de la tige souterraine à celle de la tige aérienne.

On voit, toul d'abord, les faisceaux libéro-ligneux, qui étaient réunis dans la tige souterraine, se séparer et devenir absolument semblables à ceux de la tige aérienne. Le péricycle est encore parenchymateux, mais il a acquis une plus grande épaisseur; il ne tarde pas, à un niveau plus élevé, à épaissir et à lignifier les parois de ses cellules. La structure de la tige aérienne est dès lors atteinte.

A ce premier type on peut rattacher les tiges des S. Hohenwartii Sternb., tenella Wulf., aizoides L., crassifolia, cordifolia, ciliata.

Type II. Saxifraga hirsuta L. - On retrouve dans la tige rampante l'écorce toujours très développée; les cellules de l'endoderme ont les parois minces et légèrement subérifiées; le péricycle est collenchymateux et les cellules des premières assises de la moelle le sont également.

Les faisceaux libéro-ligneux sont séparés par d'étroits rayons médullaires dont les éléments sont encore collenchymateux.

La hampe florale a une écorce bien moins épaisse, les cellules de l'endoderme ont les parois cellulosiques; mais le péricycle est composé de six à sept assises de cellules petites, à parois épaissies et lignifiées, formant à la périphérie du cylindre central un anneau scléreux assez résistant.

Les faisceaux libéro-ligneux sont séparés les uns des autres par de lirges rayons médullaires; la moelle est entièrement parenchymateuse.

Le péricycle, de collenchymateux qu'il est dans la tige rampante, ne lievient pas directement sclérenchymateux en passant dans la hampe llorale. Entre ces deux ordres de tiges il y a une région intermédiaire, très courte, dans laquelle le péricycle est devenu parenchymateux ainsi que les rayons médullaires et l'anneau externe de la moelle. A ce niveau, les faisceaux libéro-ligneux sont déjà semblables à ceux de la hampe florale et les cellules de l'endoderme n'ont plus les parois subérifiées.

La tige du $S$. umbrosa L. est la seule dont la structure se rapproche complètement de celle du S. hirsuta.

TYPE III. Saxifraga longifolia Lap. - Une structure qui peut être considérée comme intermédiaire à celles qui viennent d'être signalées 
dans le $S$. stellaris et le S. hirsuta nous est offerte dans le S. longifolia.

La tige souterraine est pourvue d'une écorce très épaisse limitée intérieurement par un endoderme dont les cellules ont les parois un peu épaissies mais non subérifiées, l'épaississement portant surtout sur les parois regardant le centre de la tige.

Quatre à six assises de cellules assez petites et parenchymateuses composent le péricycle; les faisceaux libéro-ligneux sont réunis.

Au voisinage de la hampe florale, la tige souterraine subit des modifications qui rapprochent complètement sa structure de celle de la tige rampante du $S$. hirsuta. Le péricycle et la moelle, à son pourtour, deviennent collenchymateux ; en outre, dans l'écorce, quelques-unes des assises cellulaires voisines de l'endoderme ont déjà subi, un peu auparavant, la même modification.

A partir de là, tout ce qui a été dit pour le $S$. hirsuta est applicable au S. longifolia. Les cellules du péricycle, avant de lignifier leurs parois, redeviennent, pour un moment, parenchymateuses en même temps que des rayons médullaires, très larges, séparent les faisceaux libéro-ligneux.

La structure qui vient d'être décrite se retrouve dans les tiges des S. Aizoon Jacq., Lingulata Bell., serrata Sternb., sarmentosa Linn. fil.

Type IV. Saxifraga granulata L. - La partie inférienre de la tige souterraine, placée dans le prolongement de la tige aérienne, et les coulants qui en partent, sont construits comme la tige correspondante du S. stellaris : écorce bien développée, péricycle parenchymateux, faisceaux libéro-ligneux réunis et moelle très réduite.

Mais si, examinant la tige souterraine verticale, on remonte vers la hampe florale, on ne tarde pas à remarquer que les cinq ou six dernières assises de l'écorce, l'endoderme étant excepté, ne tardent pas à épaissir et à lignifier leurs parois de manière à constituer, en dehors du cylindre central, un appareil de soutien très puissant. S'élevant toujours, on voit, à mesure que les faisceaux libéro-ligneux s'individualisent, le péricycle devenir à son tour progressivement scléreux, de telle façon que, en un certain point de cette tige, le stéréome est représenté, outre les vaisseaux, par un anneau très épais de cellules sclérifiées appartenant les unes au péricycle, les autres à l'écorce.

Chez certains individus, les cellules de l'endederme deviennent aussi scléreuses; chez d'autres c'est le petit nombre, et la majorité conserve des parois minces et cellulosiques.

Plus haut, l'anneau scléreux, formé aux dépens de l'écorce, commence 
à se modifier; ses éléments, peu à peu, redeviennent parenchymateux et, à la base de la hampe florale, il a complètement disparu.

La tige aérienne ne présente rien de particulier; sa structure est celle des tiges correspondantes qui ont déjà été étudiées.

Ici, la tige souterraine est orientée suivant la verticale et, par conséquent, supporte la tige aérienne qui n'en est que la partie supérieure et émergée; rien d'étonnant donc si le stéréome y est bien développé ; mais, dans d'autres espèces ( $S$. ajugafolia, par exemple), la mème structure se retrouve dans des tiges végétant horizontalement, dont les tissus soulenus par le sol n'ont rien à supporter.

Autour du type S. granulata viennent se grouper les S. hypnoides L. ajugefolia L., pedatifida Ehrh., geranioides L., aspera L.

Type V. Saxifraga oppositifolia. - La manière d'êtré du péricycle dans les deux ordres de tiges est ici la même que dans les S. stellaris et granulata, c'est-à-dire que, de parenchymateux qu'il est dans la tige couchée, le péricycle devient scléreux dans la tige aérienne. Mais il ne s'ensuit pas pour cela que, dans la tige couchée, le stéréome soit peu développé, il l'est au contraire considérablement; on va le voir.

Tige couchée. - Si l'on examine une pareille tige au milieu d'un entre-nœud, on y reconnaît la structure suivante : les cellules de l'exoderme sont pour la plupart scléreuses, de manière à faire partie de l'appareil de soutien; l'écorce est parenchymateuse et le péricycle l'est également.

Un peu plus haut, de légères modifications ne tardent pas à se produire : tout d'abord, tous les éléments de l'exoderme sont devenus scléreux; ensuite, aux deux extrémités du diamètre perpendiculaire à celui sur lequel se trouvent les deux faisceaux qui au plus prochain nœud se rendront dans les feuilles, les cellules de l'écorce, elles aussi, se sclérifient jusqu’à l'endoderme non compris.

Toujours en remontant, on ne tarde pas à voir les deux amas scléreux ainsi formés augmenter de puissance, s'étaler, pour ainsi dire, contre l'endoderme et prendre la forme de deux croissants, dont les cornes ont une tendance à se rejoindre au-dessus des faisceaux foliaires.

Plus haut encore, tout près du nœud, les deux pédicelles scléreux, qui rattachaient à l'exoderme ces croissants, s'étranglent et finissent par disparaître.

Au nœud, les foliaires s'infléchissent pour sortir du cylindre central et, poussant devant eux l'endoderme et le péricycle sus-jacent, ils écartent l'une de l'autre, comme des coins, les cornes des croissants scléreux. Lorsque ces faisceaux sont sortis du cylindre central, les extrémités des croissants se rejoignent, seulement alors au-dessous d'eux. 
A ce niveau, les cellules de l'assise externe des croissants, qui étaient sclérifiées, sont remplacées par des cellules à parois minces et cellulosiques.

Ces dernières cellules et celles de l'assise interne de la portion de l'écorce restée parenchymateuse ne tardent pas à se décoller sur leurs faces en contact; un espace vide se produit entre elles.

Cette rupture, grâce à laquelle les deux assises qui précédemment étaient en continuité se séparent l'une de l'autre, part, de chaque côté de la tige, du point oủ le croissant scléreux avait sa plus grande épaisseur, pour se propager, à droite et à gauche, sur tout le pourtour de la tige, qui est alors séparée en deux portions concentriques.

La portion externe, dans laquelle se trouvent les deux faisceaux foliaires qui viennent de sortir du cylindre central, fait désormais partie des deux feuilles qui à ce niveau sont insérées sur la tige; elle en forme la base.

Les deux assises cellulaires, qui limitent l'espace vide que l'on vient de voir se produire, appartiennent toutes les deux à l'épiderme de l'axe feuillé qui s'est insinué dans l'aisselle de chacune des deux feuilles; l'assise externe dépend de l'épiderme supérieur de ces feuilles, et l'assise interne de l'épiderme de la tige.

L'écorce de la tige est souvent, à ce niveau, entièrement scléreuse.

A partir de là, toutes les modifications qui viennent d'être étudiées vont se reproduire, mais en sens inverse.

Vis-à-vis des deux nouveaux faisceaux foliaires, on voit, dans l'écorce, le sclérenchyme remplacé en un point par du parenchyme; puis cette région devenue parenchymateuse s'étend, à droite et à gauche, au fur et à mesure que l'on s'éloigne du nœud. Aussi, au milieu du nouvel entre-nœud, la tige a-t elle de nouveau la structure décrite au commencement de cette étude.

Tige aérienne. - La tige aérienne offre, dans la succession des entrenœuds et des nœuds, certains détails de structure qui ne sont pas sans analogie avec ceux qui viennent d'être observés dans la tige rampante; aussi, comme pour cette dernière tige, devra-t-on examiner d'abord une section faite au milieu d'un entre-nœud. A ce niveau, l'écorce est composée uniquement de parenchyme; le péricycle est scléreux comme dans toutes les tiges qui, jusqu'ici, ont été vues.

Mais, si l'on remonte un peu vers le nœud, on voit, dans l'écorce, de petits amas de cellules scléreuses apparaître aux extrémités du diamètre perpendiculaire à celui sur lequel se trouvent les deux faisceaux qui, au noud, entreront dans les feuilles.

Un peu plus haut, ces petits amas scléreux se réunissent, et, de chaque

$$
\text { T. XXXvi. }
$$

(SÉANCES) 9 
côté de la tige, on n'en trouve plus que deux, offrant alors une certaine étendue.

Quand les faisceaux foliaires commencent à sortir du cylindre central, les deux amas scléreux, situés d'un même côté de la tige, s'écartent progressivement l'un de l'autre, en se rapprochant chacun du foliaire correspondant. Après leur départ du cylindre central, les deux amas scléreux qui, à droite et à gauche, avoisinaient chacun de ces faisceaux, n'étant plus séparés par eux, se réunissent l'un à l'autre sur la ligne médiane.

Ils ne tardent pas à s'émietter au-dessus du nœud et bientôt, leur disparition étant complète, l'écorce redevient entièrement parenchymateuse.

La tige du S. biflora All. qui, comme le S. oppositifolia, a les feuilles opposées, est construite sur ce type; celles des S. casia L. et squarrosa Sieber. ont aussi la même structure, mais avec de légères modifications provoquẻes par l'alternance des feuilles sur la tige.

Type VI. Saxifraga rotundifolia L. - Tige souterraine. - L'écorce, peu épaisse, est limitée en dedans par un endoderme dont les cellules ont les parois subérifiées.

Le péricycle est entièrement parenchymateux; les faisceaux libéroligneux ne sont pas séparés les uns des autres par des rayons médullaires, ils forment un anneau continu.

Le bois est composé de vaisseaux irrégulièrement disséminés dans un parenchyme ligneux abondant; cependant, dans la moitié interne des faisceaux ligneux, les parois d'un certain nombre des cellules du parenchyme se lignifient et deviennent en même temps tellement épaisses, que les cavités cellulaires sont réduites à un point. Ces cellules, quelquefois isolées, sont le plus souvent réunies par groupes.

Sur tout le pourtour de la moelle, on trouve également des groupes de cellules présentant les mêmes caractères.

Dans la moelle et dans le bois, les cellules ainsi sclérifiées ont, dans tous les sens, la même forme que les cellules parenchymateuses qui les entourent; elles sont, comme elles, un peu allongées dans le sens longitudinal et limitées, à chacune de leurs extrémités, par une face plane ou à peu près.

Ces amas de cellules scléreuses forment, dans la tige, des cordons, qui, on le conçoit aisément, lui donnent une grande résistance; et, cependant, cette tige, végétant horizontalement dans le sol, n'a rien à supporter.

Dans le voisinage de la tige aérienne, alors que les faisceaux libéroligneux sont déjả séparés les uns des autres par des rayons médullaires, 
THOUVENIN. - APPAR. DE SOUTIEN DANS LES TIGES DES SAXIFRAG. 131 on peut constater la disparition complète, dans le bois, des cellules scléreuses.

A la périphérie de la moelle, on ne retrouve plus les groupes de ces cellules irrégulièrement disséminés comme auparavant; ils sont situés principalement en face des rayons méduliaires, dans lesquels ils pénètrent plus ou moins.

La section de ces cellules scléreuses est alors plus considérable que celle des cellules voisines, et leurs parois, moins épaisses relativement que tout à l'heure, limitent une grande cavité cellulaire.

En même temps, le péricycle qui, jusque-là, était resté parenchymateux, commence, contre l'endoderme, à se modifier, de façon à entrer, lui aussi, dans l'appareil de soutien.

Tige aérienne. - Dans cette tige, les amas scléreux dont la présence vient d'être constatée dans les rayons médullaires ont tout à fait disparu. Le stéréome n’est plus représenté que par un anneau scléreux formé aux dépens de la moitié externe du péricycle et par les vaisseaux.

Type VII. Saxifraga tridactylites L. - Cette Saxifrage est une herbe annuelle à tige simple, dans les lieux stériles, ou plus ou moins ramifiée; à cette tige fait suite une petite racine pivotante; il n'y a pas de tige souterraine.

L'étude de la racine, prise près du collet, est nécessaire pour bien comprendre la structure de la partie basilaire de la tige; aussi est-ce par elle que nous commencerons.

La racine, au point où elle est prise, est à l'état secondaire; le cylindre central seul la compose, le liège qui s'est produit dans le péricycle ayant exfolié l'écorce.

Au centre sont les vaisseaux primaires; autour d'eux on remarque le bois secondaire, tout d'abord composé de vaisseaux irrégulièrement disséminés dans du parenchyme ligneux; mais, à sa périphérie, ce bois secondaire modifie la composition de ses éléments et n'est formé que de fibres ligneuses.

Entre les différents faisceaux du bois il n'y a pas de rayons médullaires; cette racine possède donc un stéréome formé par un étui fibreux continu, d'une épaisseur de cinq à sept assises de cellules et appartenant aux faisceaux ligneux.

Le liber, très peu épais, le péricycle et le liège sont, dans cette racine, entièrement mous.

Cette structure étant bien comprise, observons une coupe faite à la - base de la tige. On a une moelle abondante, autour de laquelle sont rangés les faisceaux libéro-ligneux séparés par des rayons médullaires; le péricycle est parenchymateux. 
Dans chaque faisceau ligneux, contre le liber, le bois est encore uniquement composé de fibres comme tout à l'heure; dans la racine et dans le prolongement de ces lames fibreuses les cellules des rayons médullaires ont épaissi et lignifié leurs parois, de telle sorte que le stéréome est encore représenté ici par un anneau fibreux continu, situé immédiatement en dedans de la circonférence sur laquelle sont disposés les faisceaux libériens.

Jusqu’à ce moment, le péricycle est parenchymateux; mais, à un niveau plus élevé, il devient scléreux et, vis-à-vis des rayons médullaires, il se confond avec l'anneau de soutien dont il vient d'être question.

A partir de là, dans un faisceau sur deux, et en alternant régulièrement, la lame fibreuse disparaît. Enfin, plus haut encore, tous les faisceaux sont débarrassés de la lame scléreuse qu'ils possédaient et la tige du S. tridactylites offre alors la structure reconnue dans toutes les tiges correspondantes étudiées jusqu'ả présent : son stéréome se compose exclusivement, outre les vaisseaux, du péricycle devenu scléreux.

Les tiges des S. controversa Sternb. et $S$. petraa (Pona) L. sont les seules, parmi les espèces étudiées, dont la structure se rapproche de S. tridactylites.

La tige dressée du $S$. petrcea émet à sa base des tiges rampantes dont la structure est celle de la tige correspondante du S. stellaris. Ces tiges, à un moment donné, redressent leur extrémité pour former des hampes florales. On voit alors, près de lá, dans la tige rampante, un anneau scléreux intra-libérien se former progressivement, pour disparaître, dans la hampe florale, de la même façon que précédemment, après que les éléments du péricycle se sont sclérifiés.

Type VIII. Saxifraga orientalis Jacq. - L'appareil de soutien fait complètement défaut dans cette Saxifrage, non seulement dans la tige souterraine, mais encore dans la tige aérienne, qui, du reste, sa structure en fait foi, est peu dressée.

Ces deux tiges ne diffèrent l'une de l'autre que par les rapports de l'écorce à la moelle et la disposition des faisceaux qui sont confluents en un anneau continu dans la tige souterraine, tandis que, dans l'autre tige, ils sont séparés par des rayons médullaires.

Le genre Saxifrage a été divisé en quinze sections par M. Engler. J'ai recherché si à chacune de ces sections correspondait un type de tige particulier, ou mieux si toutes les espèces d'une même section avaient les tiges construites sur un seul et même type.

Mais il m'a fallu reconnaître que si, dans certaines sections, les tiges des espèces qui y étaient contenues appartenaient bien à un type déter- * miné, dans d'autres il n'en élait pas ainsi. 
Toutefois, mes observations n'ayant pas porté uniquement sur la tige, mais encore sur la feuille, j’ai pu me convaincre que, malgré le petit nombre d'espèces qu'il m'a été donné d'étudier, il était possible d'établir, sans s'écarter beaucoup des sections proposées par M. Engler, une classification anatomique du genre Saxifrage.

Je me propose de traiter cette question dans un travail que j'ai entrepris sur la famille des Saxifragacées.

M. G. Bonnier fait à la Société, au nom de M. Daniel, la communication suivante :

STRUCTURE COMPARÉE DE LA FEUILLE ET DES FOLIOLES DE L'INVOLUCRE DANS LES CYNAROCÉPHALES ET GÉNÉRALITÉS SUR LES COMPOSEES, par M. L. DANIEL. (1).

\section{CyNarocéPhales.}

S'il fallait cette fois prendre la coupe seule de la base de la foliole de l'involucre pour classer les Cynarocéphales comme je l'ai fait pour les Corymbifères et les Chicoracées, mon embarras serait grand, car à cet endroit elles présentent une assez grande uniformitè de structure, au point de vue du stéréome.

C'est ainsi qu'on n'y distingue que deux types, reliés entre eux par d'insensibles transitions :

$1^{\circ} \mathrm{Ou}$ il y a seulement une bande hypodermique inférieure toujours nettement fibreuse : Serratula, Saussurea, Echinops, Chamopeuce, Galactites. Cette dernière plante établit déjà le passage au deuxième type par les quelques cellules situées sous l'épiderme supérieur et qui ont une tendance à se sclérifier;

$2^{\circ} \mathrm{Ou}$ il y a deux bandes hypodermiques; l'inférieure, la plus développée, est, comme ci-dessus, toujours formée de fibres épaisses; la supérieure, beaucoup plus variable, peut être formée par une sorte de collenchyme quelque peu scléreux, ou du parenchyme plus ou moins scléreux, ou enfin des fibres de sclérenchyme: Carduus, Cynara, Silybum, Xeranthemum, Onopordon, Cirsium, Centaurea, Lappa, Carduncellus, Carlina, Kentrophyllum, Microlonchus.

J'ai rangé ces plantes par rapport au développement croissant de la bande supérieure. C'est chez les Microlonchus, Kentrophyllum, etc., qu'elle est le plus développée et fibreuse. Il est à remarquer que les tran-

(1) Ce travail a été fait au laboratoire de Botanique de la Sorbonne, dirigé par M. G. Bonnier. 


\section{$2 \mathrm{BHL}$ Biodiversity Heritage Library}

Thouvenin, Maurice-Franc

ois. 1889. "Sur L'Appareil De Soutien Dans Les Tiges Des Saxifrages." Bulletin de la Société botanique de France 36, 125-133. https://doi.org/10.1080/00378941.1889.10830433.

View This Item Online: https://www.biodiversitylibrary.org/item/8658

DOI: https://doi.org/10.1080/00378941.1889.10830433

Permalink: https://www.biodiversitylibrary.org/partpdf/157835

\section{Holding Institution}

Missouri Botanical Garden, Peter H. Raven Library

\section{Sponsored by}

Missouri Botanical Garden

\section{Copyright \& Reuse}

Copyright Status: Public domain. The BHL considers that this work is no longer under copyright protection.

This document was created from content at the Biodiversity Heritage Library, the world's largest open access digital library for biodiversity literature and archives. Visit BHL at https://www.biodiversitylibrary.org. 